\title{
MicroRNA profiles and potential regulatory pattern during the early stage of spermatogenesis in mice
}

\author{
LUO MengMeng ${ }^{1 \dagger}$, HAO LiLi $^{2 \dagger}$, HU Fen $^{1 \dagger}$, DONG YaNan $^{1}$, GOU LiXia ${ }^{1}$, ZHANG WenDian ${ }^{3}$, \\ WANG Xin ${ }^{2}$, ZHAO YuHui ${ }^{2}$, JIA MengChun ${ }^{4 *}$, HU SongNian ${ }^{2 *} \&$ ZHANG XiuJun ${ }^{1 *}$ \\ ${ }^{1}$ College of Life Sciences, College of Psychology, Hebei United University, Tangshan 063000, China; \\ ${ }^{2}$ CAS Key Laboratory of Genome Sciences and Information, Beijing Institute of Genomics, Chinese Academy of Sciences, Beijing 100101, \\ China; \\ ${ }^{3}$ Hebei Normal University for Nationalities, Chengde 067000, China; \\ ${ }^{4}$ Department of Reproductive Endocrinology, National Research Institute for Family Planning, Beijing 100081, China
}

Received May 27, 2013; accepted June 16, 2013; published online September 28, 2014

\begin{abstract}
Spermatogenesis is a complicated and poorly understood process that relies on the precise regulation of the self-renewal and differentiation of spermatogonia. In many organisms, microRNAs (miRNAs) are involved in multiple developmental processes as critical regulators of transcriptional and post-transcriptional gene silencing. This study investigated the expression pattern of miRNAs in type B spermatogonia cells (BSc) and primary spermatocytes (PSc) of mice, using a high-throughput small RNA sequencing system. The results revealed that the expression levels of Let-7 family miRNAs were remarkably high in both cell types. Furthermore, the expression levels of miR-21, miR-140-3p, miR-103, miR-30a, miR-101b and miR-99b were decreased during the transformation from BSc to PSc. These miRNAs target vital genes that participate in apoptosis, cell proliferation and differentiation, junction assembly and cell cycle regulation. These results highlight the indispensable role of miRNAs in spermatogenesis.
\end{abstract}

spermatogenesis, miRNA profiling, post-transcriptional regulation, focal adhesion, cytoskeleton dynamic, Wnt signaling pathway

Citation: Luo MM, Hao LL, Hu F, Dong YN, Gou LX, Zhang WD, Wang X, Zhao YH, Jia MC, Hu SN, Zhang XJ. MicroRNA profiles and potential regulatory pattern during the early stage of spermatogenesis in mice. Sci China Life Sci, 2015, 58: 442-450, doi: 10.1007/s11427-014-4737-8

Spermatogenesis is a complex process in which spermatogonial stem cells form spermatozoa following mitotic, meiotic and post-meiotic phases. The process of spermatogenesis is highly sensitive to fluctuations in the environment and involves numerous endocrine and paracrine signals to coordinate the self-renewal of spermatogonial stem cells (SCCs) and spermatogonial differentiation [1]. Spermatogenesis is characterized by the phase-specific expression of many

$\dagger$ Contributed equally to this work

*Corresponding author (email: mengchunjia@163.com; husn@big.ac.cn; xiujunzhang66@126.com) genes that are exclusively expressed in spermatogenic cells. With the development and application of technologies such as gene cloning, gene expression and functional characterization, many spermatogenesis-related genes have been identified in the past few years, some of which were found to play important roles in spermatogenesis [2]. Spermatogenesis-associated genes such as cyclins, proto-oncogenes and genes for azoospermia factor, cytoskeleton, heat shock proteins, nucleoprotein transition, centrin and apoptosis are involved in highly conserved landmark events such as meiotic recombination, formation of the synaptonemal complex, sister chromatid cohesion, spermiogenesis during post- 
meiotic stages, and checkpoints and factors required for the meiotic cell cycle.

Recently, small RNA molecules, including small interfering RNAs (siRNAs), microRNAs (miRNAs, approximately 22-nt sequences) and Piwi-interacting RNAs (piRNAs, 24- to 30-nt sequences) have emerged as important regulators of gene expression at the post-transcription or translation level [3]. Several miRNAs are expressed abundantly in male germ cells, either throughout or during specific stages of spermatogenesis [4]. piRNAs, which are actively involved in retrotransposon silencing that protects the integrity of the genome, are only present in pachytene spermatocytes and round spermatids [5]. piRNAs in type A spermatogonia, pachytene spermatocytes and round spermatids were profiled by deep sequencing in a recent study, the results showed that piRNA mapping to retrotransposons, mRNAs and intergenic regions had different length distributions and were differentially regulated in spermatogenesis [6]. In a recent study, mature mouse sperm were found to be extremely enriched in a novel class of tRNA-derived small RNAs (29-34 nt), which were slightly different from the RNA found in adult testes (26-32 nt). The discovery of sperm-borne RNAs have opened the possibility of additional paternal contributions aside from providing DNA [7].

miRNAs are a family of 21-25-nt cellular non-coding RNAs that bind to the 3 '-untranslated region (UTR) of cognate mRNA through an imperfect match to repress their translation and stability $[8,9]$. This is achieved by forming a ribonucleoprotein complex termed the RNA-induced silencing complex (RISC), which contains a member of the Argonaute family [10]. Recently, the fields of research on stem cells and miRNA have converged with the identification of stem cell-specific miRNAs [11,12]. Based on their function in translation attenuation, miRNAs seem to regulate the fate and behavior of stem cells by fine-tuning the protein levels of various factors required for stem cell or niche cell functions. Several miRNAs exhibiting tissue specificity have been identified in embryonic stem cells (ESCs) and somatic stem cells (SSCs) but not in germ-line stem cells (GSCs) [13]. Notably, loss of the RISC component Dicer in germ cells or Sertoli cells has been shown to interrupt germ cell development and lead to infertility [14]. This finding highlights the importance of miRNAs in the regulation of spermatogenesis. These studies provide information about a new layer of molecules associated with the intricate mechanisms of gene regulation, including miRNAs and RISC components, suggesting that miRNAs are functionally important in spermatogenesis.

In this study, we investigated the expression pattern and functional requirements of the miRNAs in type B spermatogonia and primary spermatocytes in order to illustrate their role in the early spatiotemporal development of spermatogenesis.

\section{Materials and methods}

\subsection{Experimental cell lines}

GC-1spg and GC-2spd (ts) cells [15,16] (ATCC, USA) were maintained in Dulbecco modified Eagle medium (DMEM) containing 10\% fetal bovine serum (FBS) and 1.5 $\mathrm{g} \mathrm{L}^{-1}$ sodium bicarbonate. GC-1spg cells were created by transforming 10-day-old mouse type B spermatogonia with pSV3-neo; these cells exhibited characteristics of the stage between type B spermatogonia and primary spermatocytes. GC-2spd (ts) cells were created by transforming 6-week-old mouse spermatocytes with SV40 large T antigen; these cells lost their differentiation potential and remained arrested at the pre-meiotic stage. For simplicity, GC-1spg and GC-2spd (ts) cells were abbreviated as BSc and PSc respectively in the following results and discussion sections.

\subsection{RNA extraction, library construction and se- quencing}

Total RNA was extracted from GC-1spg and GC-2spd (ts) cell lines by Trizol (Invitrogen, USA) according to the manufacturer's instructions. The RNA samples were incubated with $10 \mathrm{U}$ DNase I (TaKaRa, China) for $30 \mathrm{~min}$ at $37^{\circ} \mathrm{C}$ to remove residual genomic DNA. The quality and quantity of the purified RNA were determined by measuring the absorbance at $260 / 280 \mathrm{~nm}\left(A_{260} / A_{280}\right)$ using a Nanodrop ${ }^{\circledR}$ ND-1000 spectrophotometer (LabTech, USA). The samples had an average RNA integrity number (RIN) value of 8.9, as determined using an Agilent 2100 Bioanalyzer (Agilent Technologies, USA). Small RNAs (18-30 nt) were gel-purified from $10 \mu \mathrm{g}$ total RNA. Adaptors were ligated to eluted RNAs successively with RNA ligase. Libraries were gel-purified after each ligation step. Small RNAs ligated with adaptors were subjected to RT-PCR (15 cycles of amplification) to produce sequencing libraries. PCR products were purified on a non-denaturing acrylamide gel and sequenced for 50 cycles on Illumina HiSeq 2000 Sequencer (BGI, China).

\subsection{Analysis of sequencing data}

Each tunnel generates millions of raw reads with a sequencing length of $49 \mathrm{bp}$. Sequencing data were mainly analyzed using the Short Oligonucleotide Alignment Program (SOAP) [17]. After eliminating low-quality reads, accurately clipping the adaptor sequences and removing reads whose lengths were smaller than $18 \mathrm{nt}$ with the aid of a dynamic Perl programming algorithm, clean reads were mapped to mouse genome mm9. Reads were then screened against the Rfam and NCBI GenBank databases and classified into different types such as small RNAs, mRNAs and other types (i.e., rRNA, tRNA, scRNA, snRNA, snoRNA, repeat-associated small RNAs and so on). Known miRNAs 
such as small RNAs that perfectly matched the miRBase (version 18.0) mouse miRNA precursor sequences and those identified as known miRNA editing polymorphisms were annotated and calculated to only one category according to priority.

\subsection{Detection of differentially expressed miRNAs}

We compared the expression profiles of the identified miRNAs in both samples. The expression level was normalized using TPM for calculating the fold change between GC-1spg and GC-2spd (ts) cell lines. Differentially expressed miRNAs were identified using a rigorous algorithm as described previously [18]. Fold change between the two samples were calculated, if the $\log 2$ value of the fold change between two samples was greater than 1 or less than -1 , and the $P$-value was less than 0.05 , the miRNA was considered to be a potentially differentially expressed miRNA.

\subsection{Prediction of miRNA target genes and enrichment analysis of mouse KEGG pathways}

The predicted target genes of all mouse miRNAs were downloaded from the miRWalk database, the prediction results combine comparative analysis of five prediction programs-DIANA-mT, miRanda, miRDB, RNA22 and TargetScan [19] and parameters for searching the miRWalk were as follows: Gene region 3'-UTR, min seed length 7, $P$-Value 0.05, Transcript longest. Our own Perl script was used to filter the combination results. Enrichment analysis of KEGG pathway revealed the main pathways in which the candidate target genes are involved. This analysis identifies significantly enriched metabolic pathways or signal transduction pathways in which the candidates of target genes are involved by comparing the genes with the whole reference gene background. Two-sided Fisher's exact test corrected by multiple testing and the $\chi^{2}$ test were used to classify the pathway category. FDR was used to correct the $P$ value, and only pathway categories with corrected $P$-value $\leqslant 0.05$ were selected as enriched ones.

\subsection{Stem-loop RT-PCR}

Total RNA was extracted from GC-1spg and GC-2spd using the same methods as mentioned in RNA extraction and library construction. For the RT-PCR, the reaction mixture contained purified total RNA, cell lysate or heat-treated cells as the RNA samples, $50 \mathrm{nmol} \mathrm{L}^{-1}$ stem-loop RT primer, $1 \times$ RT buffer (Epicentre, USA), $0.25 \mathrm{mmol} \mathrm{L}^{-1}$ of each

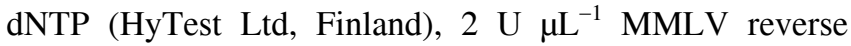

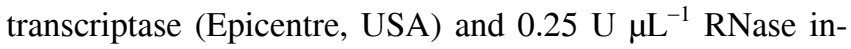
hibitor (Epicentre, USA). The 7.5- $\mu \mathrm{L}$ reaction mixtures were incubated in an GeneAmp PCR System 9700 (Applied Biosystems, USA) in a 96- or 384-well plate for $30 \mathrm{~min}$ at $16^{\circ} \mathrm{C}, 40 \mathrm{~min}$ at $42^{\circ} \mathrm{C}, 5 \mathrm{~min}$ at $85^{\circ} \mathrm{C}$ and then held at $4^{\circ} \mathrm{C}$. All reverse transcriptase reactions, including no-template controls and RT minus controls, were run in duplicate.

Real-time PCR was performed using a standard TaqMan ${ }^{\circledR}$ PCR kit protocol on an Applied Biosystems 7900HT Sequence Detection System (Applied Biosystems). The $10-\mu \mathrm{L}$ PCR mixture consisted of $0.67 \mu \mathrm{L}$ of RT product, $1 \times$ TaqMan ${ }^{\circledR}$ Universal PCR Master Mix (Applied Biosystems), $0.2 \mu \mathrm{mol} \mathrm{L}^{-1}$ TaqMan $^{\circledR}$ probe, $1.5 \mu \mathrm{mol} \mathrm{L}^{-1}$ forward primer and $0.7 \mu \mathrm{mol} \mathrm{L} \mathrm{L}^{-1}$ reverse primer. The reactions were incubated in a 384 -well plate at $95^{\circ} \mathrm{C}$ for $10 \mathrm{~min}$, followed by 40 cycles of $95^{\circ} \mathrm{C}$ for $15 \mathrm{~s}$ and $60^{\circ} \mathrm{C}$ for $1 \mathrm{~min}$. All reactions were run in triplicate. The threshold cycle $\left(C_{\mathrm{t}}\right)$ was defined as the fractional cycle number at which fluorescence passes the fixed threshold. The average $C_{\mathrm{t}}$ value was calculated for each sample using the $2^{-\Delta \Delta C_{\mathrm{t}}}$ method, normalized to U6, and expressed relative to spermatogonia [20].

\section{Results}

\subsection{Overview of small RNA sequencing results}

After preparation of small RNA sequencing libraries, Illumina high-throughput sequencing was used to characterize all small RNA species in both BSc and PSc. Initially, we obtained 17209025 and 15352366 raw reads for BSc and PSc respectively. After excluding reads containing ambiguous bases, adaptor contaminants and short reads, 13512566 $(79.17 \%)$ and $9283464(60.95 \%)$ clean reads were left for BSc and PSc respectively. Clean reads from both BSc and PSc showed exclusive length distribution with a distinct peak at 19-22 nt (Figure 1A and B). In total, 9914986 $(73.38 \%, \mathrm{BSc})$ and $6633665(71.46 \%, \mathrm{PSc})$ clean reads could be mapped to the mouse $\mathrm{mm} 9$ genome. In BSc, $6285296(46.51 \%)$ clean reads were identified as miRNAs, while this number was 3126965 (33.68\%) in PSc. Reads that could not be classified to any known RNA groups formed a considerable proportion of the annotation results at 2116886 BSc (15.67\%) and 1126311 PSc (12.13\%; Figure $2 \mathrm{~A}, \mathrm{~B}$ and Table S1 in Suppporting Information).

\subsection{Abundant expression of mmu-let-7 family in both BSc and PSc}

The expression value of each known miRNA was normalized by TPMs (transcripts per million). Of 717 miRNAs checked, 322 miRNAs were expressed in at least one of the two cell lines. The results revealed that in BSc, $42.2 \%$ (136) of the miRNAs were poorly expressed (with TPMs $<10$ ), $54.7 \%$ (176) miRNAs were moderately expressed (with TPMs $10-10000$ ), and only $3.1 \%$ (10) miRNAs were abundantly expressed (with TPM $>10000$ ). These 10 miRNAs accounted for $75.6 \%$ (4925373) of all miRNA reads. In PSc, the number of poorly, moderately and abundantly expressed miRNAs are $163(50.6 \%), 154(47.8 \%)$ and $5(1.6 \%)$ re- 
spectively, the highly expressed miRNA reads accounting for $65.9 \%$ (2181566) of all miRNA reads (Table S2).

Interestingly, reads mapping to mmu-let-7a, mmu-let-7b, mmu-let-7c, mmu-let-7d, mmu-let-7e, mmu-let-7f, mmulet-7g and mmu-let-7i accounted for $57.2 \%$ (3952243) and $66.8 \%$ (2320407) of all miRNA reads in BSc and PSc, respectively (Table S2), which means that most members in mmu-let7 family play fundamental roles in maintaining status of these two cell lines. Among the eight mmu-let-7 family members detected, mmu-let-7g and mmu-let-7i were significantly down-regulated in PSc, indicating they may exert regulatory effect during the transformation from $\mathrm{BSc}$ to PSc (Table 1). Let-7 family miRNAs are thought to be master regulators of cell proliferation pathways [21], our findings in BSc and PSc show that let-7 family may play indispensable role in GSC development.

\subsection{Differentially expressed miRNAs distinct from other types of stem cells}

Totally, 179 miRNAs were detected as differentially expressed, of which 142 miRNAs were down regulated and 37 were up regulated in PSc (Figure 3, Table S2). We intended to determine which miRNAs, especially those that have not yet been reported, play a major role in GSC (germ stem cell) development. Forty miRNAs with TPM>100 in either

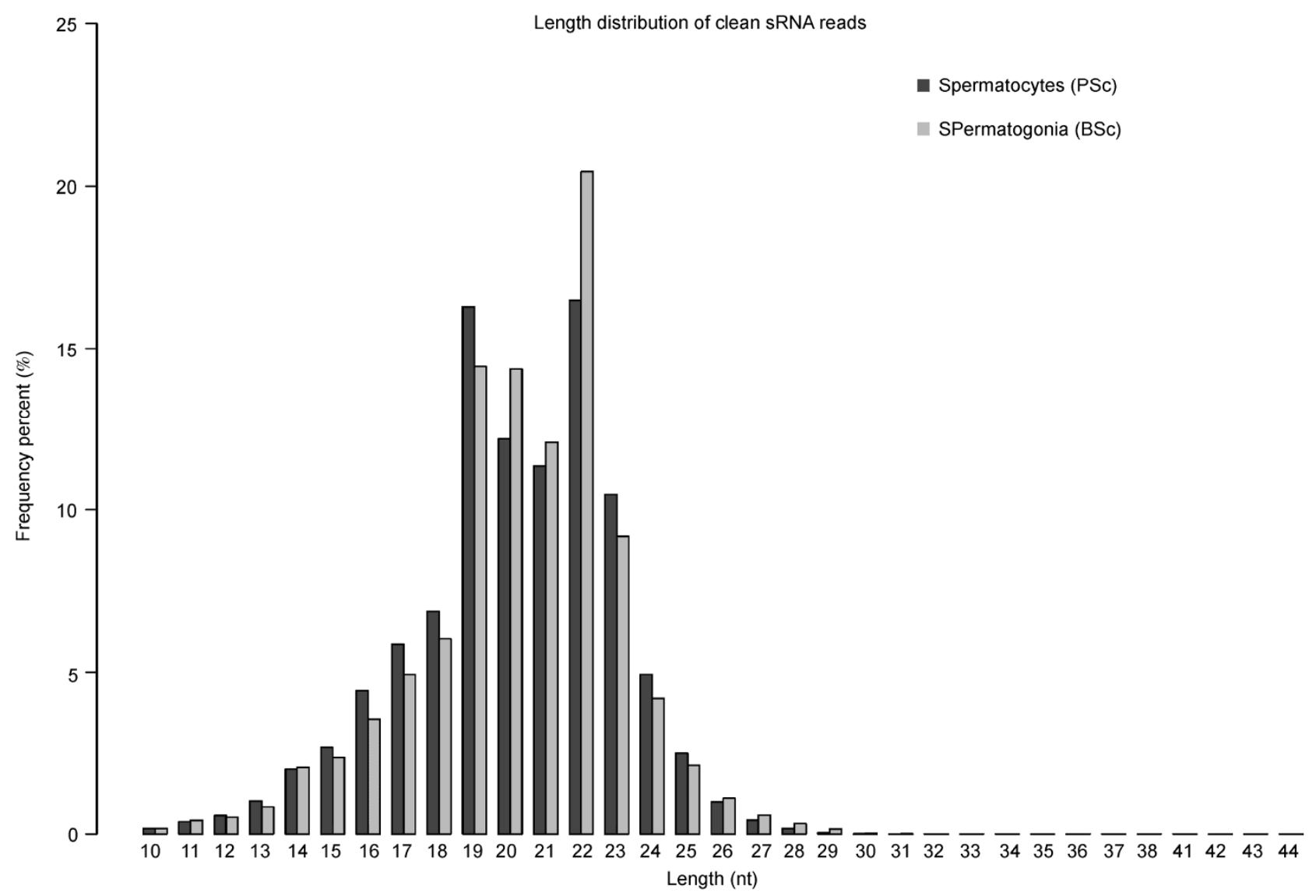

Figure 1 Length distribution of all clean small RNA reads of type B spermatogonia cells (BSc) and primary spermatocytes (PSc).

Table 1 Let-7 family expression among BSc and PSc

\begin{tabular}{lrrccc}
\hline miR-name & PSc (TPM) & BSc (TPM) & $\begin{array}{c}\text { Fold-change } \\
(\log 2 \text { BSc/PSc) }\end{array}$ & $P$-value & Sig-label \\
\hline mmu-let-7a & 44323.7567 & 43445.3382 & -0.02887878 & $7.58 \times 10^{-23}$ \\
mmu-let-7b & 48413.7171 & 38769.8384 & -0.32048115 & 0 \\
mmu-let-7c & 102487.2828 & 124275.4337 & 0.27809623 & 0 & $1.51 \times 10^{-97}$ \\
mmu-let-7d & 8888.6002 & 9752.3298 & 0.13379068 & 0 & 0 \\
mmu-let-7e & 19016.5007 & 22532.3599 & 0.24474663 & 0 & $* *$ \\
mmu-let-7f & 20753.5678 & 36147.3905 & 0.80053213 & 0 & $* *$ \\
mmu-let-7g & 2927.3556 & 7043.7399 & 1.26674363 & 1.7444092 & 0 \\
mmu-let-7i & 3139.7763 & 10520.0596 & & & 0 \\
\hline
\end{tabular}



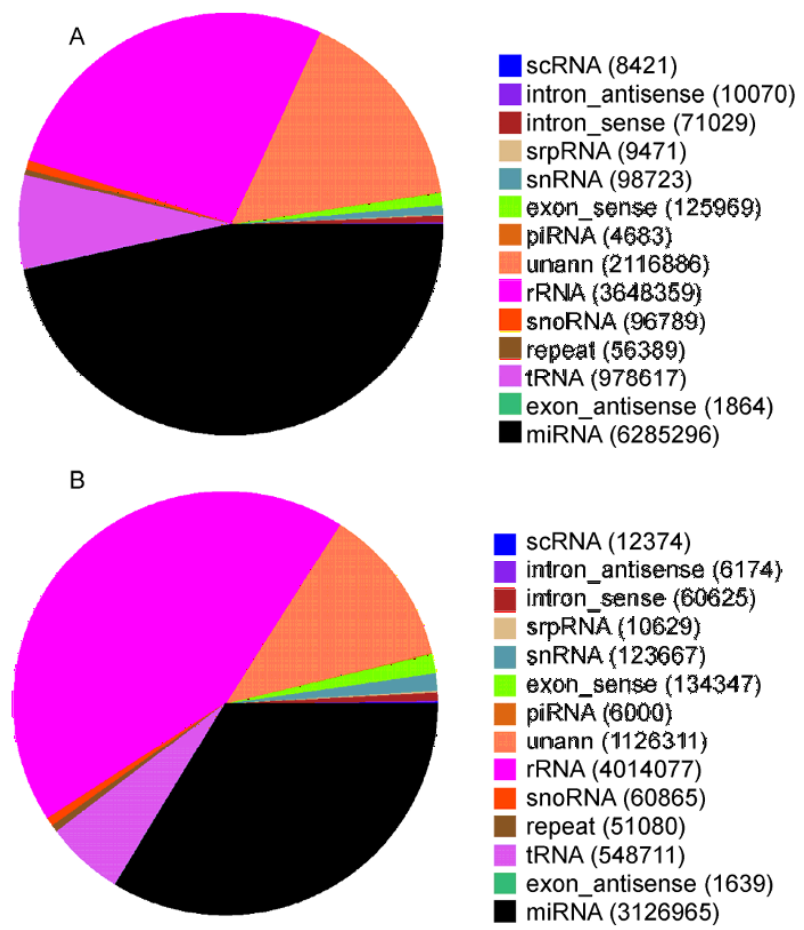

Figure 2 Annotation of RNA classification on all clean small RNA reads in both type B spermatogonia cells (BSc) (A) and primary spermatocytes (PSc) (B).

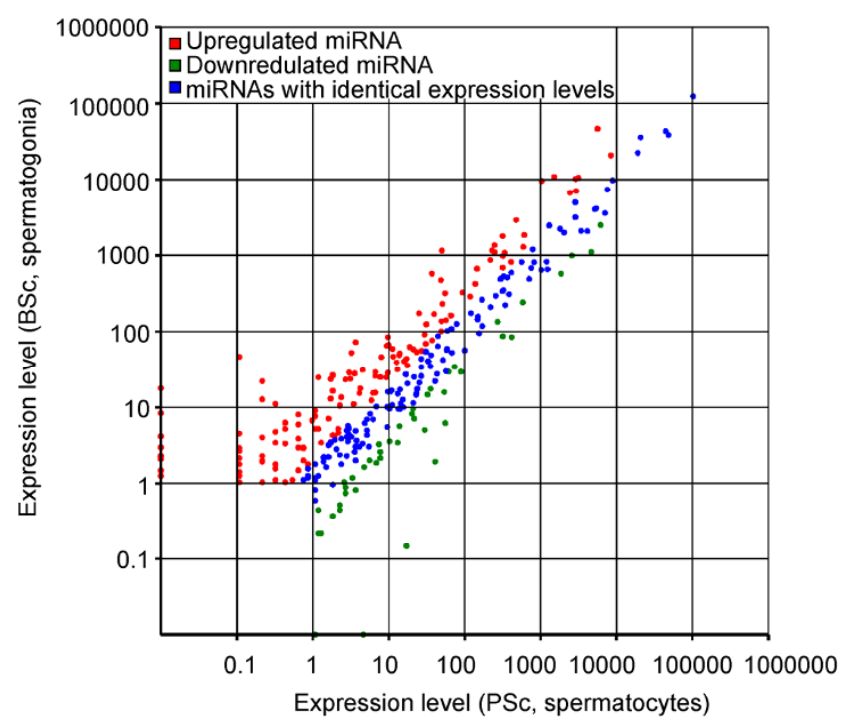

Figure 3 Scatter plot of all detected miRNAs' expression levels of type B spermatogonia cells (BSc) and primary spermatocytes (PSc).

BSc or PSc were termed as significantly differentially expressed miRNA (SDE miRNA). Among these $40 \mathrm{SDE}$ miRNAs, 10 were with TPM>2000 in either of the cell lines, they were considered to play important roles during early GSC development (Table S2). Among them, mmu-let-7i, mmu-miR-140-3p, mmu-miR-21, mmu-miR-103, mmumiR-30a and mmu-miR-101b were expressed with TPM of almost near or greater than 10000 in BSc but were remark- edly lower in PSc than in BSc.

Currently, no miRNAs dedicated specially to GSCs have been discovered. The miR-290-295, miR-17-92 and miR-15b-16 clusters have been reported to be vital for self-renewal of ESCs in mice, while miR-22 was found to be vital for differentiation of ESCs [12,22]. Of these three clusters, miR-290-295 cluster was not expressed in either BSc or PSc in our study (Table S2). miRNAs in the miR-17-92 cluster were down-regulated during the transition from BSc to PSc, albeit to different extents. Except for mmu-miR-92a, members of the miR-17-92 cluster were all expressed with TPMs $<1000$ (Table S2). Among miRNAs of the miR-15b-16 cluster, miR-15b was up-regulated by nearly two-fold in PSc, but miR-16 showed no apparent difference. MiR-22 also showed no difference between BSc and PSc (Table S2).

\subsection{Functional enrichment analysis of target genes of SDE miRNAs and candidate marker genes regulated by SDE miRNAs}

We focused on the target genes of SDE miRNAs and explored their potential roles during germ stem cell development. Because different genes usually cooperate with each other to play different roles in biological functions, pathway-based analysis could aid in obtaining a better understanding of the biological function of genes. Based on the target prediction results of all SDE miRNA on the miRWalk website, we checked the combined prediction results of five programs - DIANA-mT, miRanda, miRDB, RNA22 and TargetScan. We call it a miRNA-target pair if the targeting relationship is supported by at least two programs. Using this strict filtering conditions, 40 SDE miRNA were predicted to target 12835 genes (Table S3). All these target genes were checked using our published DGE (digital gene expression) data generated from the same cell lines [23] to filter out genes which were not differentially expressed, or which showed the same expression pattern as corresponding miRNAs. 1437 genes passed the filtering, which means that these DEGs are highly prone to be regulated by corresponding miRNAs and they were termed miRNA-targetedDEGs (Table S3).

Through functional enrichment analysis of KEGG pathways, we found that the 1437 miRNA-targeted-DEGs were enriched in four pathways they were pathways in cancer, apoptosis, gap junction and focal adhesion respectively (corrected $P<0.05$; Table 2). Among these four pathways, the DEGs detected between BSc and PSc were found to be also enriched in three of them-pathways in cancer, apoptosis and focal adhesion, which indicates that these functionally important pathways during the transition from BSc to PSc are potentially affected or regulated by SDE miRNA.

Among the target genes in the four enriched pathways, FGF7, FGF13 and WNT10A in mmu05200-pathways in cancer, were reported as candidate markers which are dif- 
ferentially expressed on both mRNA and protein levels and can discriminate BSc from PSc [23]. Based on the target prediction results, FGF7 was up-regulated in PSc and was targeted by mmu-miR-503 and mmu-miR-340-5p. FGF13 and WNT10A were both down-regulated in PSc, FGF13 was targeted by mmu-miR-143 and WNT10A was targeted by mmu-miR-206 and mmu-miR-143 (Table 3).

\subsection{Experimental validation of potential miRNA markers}

As shown above, of the 40 significantly differentially expressed miRNAs identified in this study, mmu-let-7i, mmumiR-140-3p, mmu-miR-21, mmu-miR-103, mmu-miR$101 \mathrm{~b}$ and mmu-miR-30a were expressed with TPM values of almost or greater than 10000 in BSc but with extremely low TPM in PSc. Stem-loop reverse transcription (RT)polymerase chain reaction (PCR) analysis was used to verify the expression levels of these six miRNAs (Table S4). The RT-PCR results of the six miRNAs correlated well with the sequencing results (Figure S1), indicating that these six miRNAs were truly dramatically changed and they may be used as candidate miRNA markers to distinguish BSc from PSc.

\section{Discussion}

\subsection{Germ cell lines as models to study the early stage of spermatogenesis}

Study of gametogenesis and meiotic process that is funda- mental for reproduction and the maintenance of genetic diversity require establishing mammalian germ-cell lines capable of differentiation in vitro. Hofmann et al. [16] had reported the immortalization of all the cell types contributing to a developing seminiferous tubule in mouse testis using the SV40 large T antigen. These cell types include 16 peritubular, 22 Leydig, 8 Sertoli, and 1 germ cell line, the germ cell line (GC-1spg) was established that corresponds to a stage between spermatogonia type $\mathrm{B}$ and primary spermatocyte, based on its characteristics in phase contrast and electron microscopy. These four immortalized cell types are able to reaggregate and form structures resembling two-dimentional spermatogenic tubules in vitro. This means that these immortalized cells can exert similar functions as they are in vivo. GC-2spd(ts) were obtained by mouse primary germ cells enriched in preleptotene spermatocytes cotransfected with the plasmid pSV3neo containing the LTAg gene and the plasmid LTRp53cG9 containing the temperature sensitive p53 gene ((ts)p53) [15]. At permissive temperature of 37 degrees, the GC-2spd (ts) cell line generates cells with a haploid DNA content and morphologic and biochemical features of round spermatids. Since both GC-1spg and GC-2spd (ts) are able to proliferate over long periods of time and to differentiate in vitro, they would greatly facilitate the identification and characterization of the factors that induce germ-cell proliferation or meiosis. It is appropriate that we used these two cell lines to test the roles played by miRNA during early stage of mouse spermatogenesis.

Table 2 Enriched pathways of the target genes of SDE miRNAs

\begin{tabular}{|c|c|c|c|}
\hline Term & $P$-Value & Genes & Bonferroni correction \\
\hline mmu05200: Pathways in cancer & $7.87 \times 10^{-6}$ & $\begin{array}{c}E 2 F 1, F G F 5, F G F 7, \text { PDGFB, PDGFA, FGF11, FGF13, GLI3, MMP2, } \\
C C N E 2, F O S, \text { RALA, FAS, WNT6, AKT2, WNT10A, WNT1OB, } \\
\text { RUNX1T1, CDK6, PIAS2, WNT11, LAMC2, LAMC1, WNT9A, XIAP, } \\
P M L, E G L N 3, \text { NFKBIA, EGLN2, PIK3R5, AXIN2, RUNX1, TRAF6, } \\
\text { FIGF, FN1, APC, EPAS1, BRCA2, IGF1, SMAD2, BIRC2, KITL, FZD7, } \\
\text { DVL1, NRAS, ITGA6, RASSF1, JAK1, WNT7A }\end{array}$ & 0.0014 \\
\hline mmu04210: Apoptosis & $1.04 \times 10^{-4}$ & $\begin{array}{c}\text { IRAK1, XIAP, NFKBIA, ENDOD1, CAPN2, BIRC2, PRKAR2B, } \\
\text { TNFRSF1A, IRAK3, MYD88, TNFRSF1OB, PRKAR1B, PPP3CB, } \\
\text { PIK3R5, PRKACA, PRKACB, FAS, NGF, AKT2 }\end{array}$ & 0.0180 \\
\hline mmu04540: Gap junction & $2.88 \times 10^{-4}$ & $\begin{array}{c}\text { GNAI3, GNAI2, PDGFB, ADCY7, GNAI1, PDGFA, GNA11, PRKG2, } \\
\text { LPAR1, GRM1, NRAS, ADCY9, TUBA4A, PRKACA, GNAS, PDGFD, } \\
\text { PRKACB, TUBB3 }\end{array}$ & 0.0491 \\
\hline mmu04510: Focal adhesion & $2.98 \times 10^{-4}$ & $\begin{array}{c}\text { 2900073G15RIK, XIAP, PDGFB, PDGFA, PIP5K1C, ELK1, ITGB3, } \\
\text { DOCK1, COL6A1, PIK3R5, ZYX, PAK1, PDGFD, THBS1, FIGF, } \\
\text { COL11A1, AKT2, FN1, FLT1, ROCK2, IGF1, CAPN2, BIRC2, COL5A2, } \\
\text { ITGA6, RAP1A, RAP1B, LAMC2, LAMC1, PARVB, MYLK }\end{array}$ & 0.0508 \\
\hline
\end{tabular}

Table 3 Candidate marker genes which can discriminate type B spermatogonia cells (BSc) and primary spermatocytes (PSc) and their potential corresponding miRNA regulators

\begin{tabular}{ccc}
\hline DEG marker & DEG pattern & miRNA \\
\hline Fgf7 & $\uparrow$ & mmu-miR-503, mmu-miR-340-5p \\
Fgf13 & $\downarrow$ & mmu-miR-143 \\
Wnt10a & $\downarrow$ & mmu-miR-206, mmu-miR-143 pattern \\
$\uparrow$
\end{tabular}




\subsection{Highly expressed mmu-let-7 family in BSc and PSc implies their involvement in early stage of spermatogen- esis}

In this study, we found that seven members of the mmu-let7 miRNA family were the most abundantly expressed miRNAs in both BSc and PSc; in particular, mmu-let-7a-f and let-7i were the most abundantly expressed miRNAs. mmu-let-7a-f accounted for a large proportion of all the miRNAs $(80 \%$ in the case of BSc and $82.3 \%$ in PSc), mmu-let-7g and mmu-let-7i showed significantly differential expression pattern between BSc and PSc. As one of the first two known miRNAs (the other one is lin-4) [24,25] conserved in invertebrates and vertebrates, let- 7 has been extensively studied. It controls the timing of cell cycle exit and terminal differentiation in Caenorhabditis elegans and is poorly expressed or deleted in human cancers and cancer stem cells, acting as a tumor suppressor [26]. As one of the four genes involved in induced pluripotent stem (iPS) cell reprogramming [27], LIN28 expression is the opposite to that of mature let-7 [28]. Let-7 might enhance its own level by repressing its negative regulator LIN28 [29-31]. Microarray analysis has revealed many genes regulating cell cycle and cell proliferation that are responsive to alterations in let-7 expression levels, including cyclin A2, CDC34, Aurora $A$ and B kinases (STK6 and STK12), E2F5 and CDK8 [32]. Subsequent experiments have confirmed the direct effects of some of these genes, such as CDC25A and CDK6 [26]. Let-7 also inhibited several components of the DNA replication mechanism, transcription factors and even some tumor suppressor genes and checkpoint regulators [32]. Indeed, let-7 family members are also abundant in the testes [33] and spermatozoa [34], suggesting that let-7 family members may contribute to the regulation of the male germ cell lineage. The extreme activity of most let-7 family members in both BSc and PSc demonstrates their importance to early stage of spermatogenesis. Additionally, our findings demonstrate that mmu-let-7g and mmu-let-7i may be particularly crucial during differentiation from BSc to PSc, as only their expression level dropped sharply across these two cell lines.

\subsection{Potential marker miRNAs may distinguish BSc from PSc}

The expression levels of mmu-miR-21, mmu-miR-140-3p, mmu-miR-103, mmu-miR-30a and mmu-miR-101b were close to those of the let-7 family members and showed significant decreases during the transition from BSc to PSc, indicating their active involvement in the transition from BSc to PSc. The significant expressional difference between the two cell lines suggests that these miRNAs may be key regulators of the transition from BSc to PSc. These miRNAs were down-regulated to regulate the expression of corresponding target genes which promote the transformation from mitotic to meiotic state. miR-21 was one of the first mammalian miRNAs to be identified, and it is active in many processes such as apoptosis [35], development [36], oncology [37] and stem cells [14]. Furthermore, miR-140 has been shown to be essential for normal endochondral bone development and proliferation [38], while miR-103 is linked to insulin sensitivity [39] and intestinal cell proliferation [40]. Recently, miR-30a has been found to suppress tumor growth in colon carcinoma by targeting DTL gene [41]. MiR-101b cooperates with let-7 miRNAs in tumors to inhibit cell proliferation [42], and it also participates in the FGF-2-regulated cell proliferation, migration and angiogenesis through an NDY1/KDM2B-miR-101-EZH2 pathway [43]. Among the six candidate miRNA markers, miR-21 has been found to be involved in the differentiation of ESCs [22] and to target the Nanog and Sox 2 genes [44]. One report has shown that miR-103 cooperated with miR-107 in hematopoiesis from SSCs [45]; however, no studies have described the involvement of any of the other three miRNAs in stem cell development.

These potential marker miRNAs are related to developmental processes, including proliferation and differentiation. As stem cell specific miRNAs showed distinct profiles in specific cell types or during specific stages of differentiation [13], miRNAs that were differentially expressed in BSc and PSc but distinct from the other known stem cell-related miRNAs can be regarded as the candidate marker miRNAs that may distinguish spermatogonia from spermatocytes. Further studies are required to elucidate the specific roles of these miRNAs in the early stage of spermatogenesis.

\subsection{Interconnected relationship between differentially expressed miRNAs and their target genes involved in the transition from BSc to PSc}

miRNAs perform their function by targeting corresponding mRNAs coding for important proteins in vital pathways of the developmental process, and regulating their expression levels spatiotemporally. Many of the genes and transcription factors potentially involved in spermatogenesis have already been identified and most are essential for regulating the early mitotic phase of spermatogenesis [46]. In this study, we compared the miRNA expression between BSc and PSc, the target genes of significantly differentially expressed miRNAs should be closely related with the regulation of spermatogenesis. Many known targets of these miRNAs and their functions have been studied. The known target genes barely participate explicitly in spermatogenesis-related pathways, and the knowledge of these genes is restricted due to the poor understanding of the relationship between miRNAs and the development of GSCs.

Using DGE (digital gene expression tag profiling) analysis on the same cell lines used in this study, Zhang et al. [23] have found that pathways related to focal adhesion, pathways in cancer, axon guidance, apoptosis and so on are rel- 
evant to early stage of spermatogenesis. These pathways may cooperate with each other to regulate and promote the differentiation from BSc to PSc. Also, some candidate marker genes which are differentially expressed were experimentally tested further on protein expression level. In this study, miRNA and target prediction results indicated that FGF7, FGF13 and WNT10A may be regulated by some SDE miRNAs, further functional study is needed to test this conjecture. Pathway enrichment analysis of SDE miRNAs' targets indicated that miRNAs may play important post-transcriptional regulatory roles during the transformation from BSc to PSc, especially on pathway in cancer, apoptosis, gap junction and focal adhesion signaling pathways. Except for gap junction pathway, the other three pathways are all significantly affected during the transition from BSc to PSc, which means that these SDE miRNAs may cooperate with each other to involve in the regulation network. Real relationship between these SDE miRNA or candidate marker miRNA and the functional pathways needs further investigation.

The authors declare that they have no conflict of interest.

This work was supported by the National Natural Science Foundation of China (81170616, 81072093, 30671092, 81302323, 31100915), the Natural Science Foundation of Hebei Province (C2014209140, C2009001260, C2012401039, H2013209194, C2013209024) and the Scientific and Technical Supporting Programs of Hebei Province (10276109D).

1 de Rooij DG. Proliferation and differentiation of spermatogonial stem cells. Reproduction, 2001, 121: 347-354

2 Zhou Q, Griswold MD. Regulation of spermatogonia. Stembook, 2008

3 Tolia NH, Joshua TL. Slicer and the argonautes. Nat Chem Biol, 2007, 3: 36-43

4 Maatouk DM, Loveland KL, Mcmanus MT, Moore K, Harfe BD. Dicer1 is required for differentiation of the mouse male germline. Biol Reprod, 2008, 79: 696-703

5 Lin H. piRNAs in the germ line. Science, 2007, 316: 397

6 Gan HY, Lin XW, Zhang ZQ, Zhang W, Liao SY, Wang LX, Han CS. piRNA profiling during specific stages of mouse spermatogenesis. RNA, 2011, 17: 1191-1203

7 Peng H, Shi J, Zhang Y, Zhang H, Liao S, Li W, Lei L, Han C, Ning L, Cao Y, Zhou Q, Chen Q, Duan E. A novel class of tRNA-derived small RNAs extremely enriched in mature mouse sperm. Cell Res, 2012, 22: 1609-1612

8 Rana TM. Illuminating the silence: Understanding the structure and function of small RNAs. Nat Rev Mol Cell Bio, 2007, 8: 23-36

9 Kim VN, Nam JW. Genomics of microRNA. Trends Genet, 2006, 22 $165-173$

10 Tijsterman M, Plasterk RH. Dicers at RISC; the mechanism of RNAi. Cell, 2004, 117: 1-3

11 González-González E, López-Casas PP, Del Mazo J. Gene silencing by RNAi in mouse Sertoli cells. Reprod Biol Endocrinol, 2008, 6: 29

12 Hayashi K, Chuva de Sousa Lopes SM, Kaneda M, Tang F, Hajkova P, Lao K, O'Carroll D, Das PP, Tarakhovsky A, Miska EA, Surani MA. microRNA biogenesis is required for mouse primordial germ cell development and spermatogenesis. PLoS One, 2008, 3: e1738

13 Gangaraju VK, Lin HF. MicroRNA: Key regulators of stem cells. Nat Rev Mol Cell Biol, 2009, 10: 116-125

14 Niu Z, Goodyear SM, Rao S, Wu X, Tobias JW, Avarbock MR,
Brinster RL. MicroRNA-21 regulates the self-renewal of mouse spermatogonial stem cells. Proc Natl Acad Sci USA, 2011, 108: 12740-12745

15 Hofmann MC, Hess RA, Goldberg E, Millan JL. Immortalized germ cells undergo meiosis in vitro. Proc Natl Acad Sci USA, 1994, 91: 5533-5537

16 Hofmann MC, Narisawa S, Hess RA, Millan JL. Immortalization of germ cells and somatic testicular cells using the SV40 large T antigen. Exp Cell Res, 1992, 201: 417-435

17 Li R, Li Y, Kristiansen K, Wang J. SOAP: Short oligonu- cleotide alignment program. Bioinformatics, 2008, 24: 713-714

18 Audic S, Claverie JM. The significance of digital gene expression profiles. Genome Res, 1997, 7: 986-996

19 Dweep H, Sticht C, Pandey P, Gretz N. Mirwalk-database: Prediction of possible miRNA binding sites by "walking" the genes of three genomes. J Biomed Inform, 2011, 44: 839-847

20 Chen C, Ridzon DA, Broomer AJ, Zhou Z, Lee DH, Nguyen JT, Barbisin M, Xu NL, Mahuvakar VR, Andersen MR, Lao KQ, Livak KJ, Guegler KJ. Real-time quantification of microRNAs by stemloop RT-PCR. Nucleic Acids Res, 2005, 33: e179

21 Boyerinas B, Park SM, Hau A, Murmann AE, Peter ME. The role of let-7 in cell differentiation and cancer. Endocri Relat Cancer, 2010, 17: $19-36$

22 Houbaviy HB, Murray MF, Sharp PA. Embryonic stem cell-specific microRNAs. Dev Cell, 2003, 5: 351-358

23 Zhang X, Hao L, Meng L, Liu M, Zhao L, Hu F, Ding C, Wang Y, He B, Pan Y, Fang W, Chen J, Hu S, Jia M. Digital gene expression tag profiling analysis of the gene expression patterns regulating the early stage of mouse spermatogenesis. PLoS One, 2013, 8: e58680

24 Rougvie AE. Control of developmental timing in animals. Nat Rev Genet, 2001, 2: 690-701

25 Lee RC, Feinbaum RL, Ambros V. The C. elegans heterochronic gene lin-4 encodes small RNAs with antisense complementarity to lin-14. Cell, 1993, 75: 843-854

26 Johnson CD, Esquela-Kerscher A, Stefani G, Byrom M, Kelnar K, Ovcharenko D, Wilson M, Wang X, Shelton J, Shingara J, Chin L, Brown D, Slack FJ. The let-7 microRNA represses cell proliferation pathways in human cells. Cancer Res, 2007, 67: 7713-77122

27 Yu J, Vodyanik MA, Smuga-Otto K, Antosiewicz-Bourget J, Frane JL, Tian S, Nie J, Jonsdottir GA, Ruotti V, Stewart R, Slukvin II, Thomson JA. Induced pluripotent stem cell lines derived from human somatic cells. Science, 2007, 318: 1917-1920

28 Viswanathan SR, Daley GQ, Gregory RI. Selective blockade of microRNA processing by lin28. Science, 2008, 320: 97-100

29 Newman MA, Thomson JM, Hammond SM. Lin-28 interaction with the let-7 precursor loop mediates regulated microRNA processing. RNA, 2008, 14: 1539-1549

30 Piskounova E, Viswanathan SR, Janas M, LaPierre RJ, Daley GQ, Sliz P, Gregory RI. Determinants of micro processing inhibition by the developmentally regulated RNA-binding protein lin28. J Biol Chem, 2008, 283: 21310-21314

31 Moss EG, Tang L. Conservation of the heterochronic regulator lin-28, its developmental expression and microRNA complementary sites. Dev Bio, 2003, 258: 432-442

32 Johnson SM, Grosshans H, Shingara J, Byrom M, Jarvis R, Cheng A, Labourier E, Reinert KL, Brown D, Slack FJ. Ras is regulated by the let-7 microRNA family. Cell, 2005, 120: 635-647

33 Gillis AJ, Stoop HJ, Hersmus R, Oosterhuis JW, Sun Y, Chen C, Guenther S, Sherlock J, Veltman I, Baeten J, van der Spek PJ, de Alarcon P, Looijenga LH. High-throughput microRNAome analysis in human germ cell tumours. J Pathol, 2007, 213: 319-328

34 Martins RP, Krawetz SA. RNA in human sperm. Asian J Androl, 2005, 7: 115-120

35 Seike M, Goto A, Okano T, Bowman ED, Schetter AJ, Horikawa I, Mathe EA, Jen J, Yang P, Sugimura H, Gemma A, Kudoh S, Croce CM, Harris CC. miR-21 is an EGFR-regulated anti-apoptotic factor in lung cancer in never-smokers. Proc Natl Acad Sci USA, 2009, 106: 12085-12090 
36 Sayed D, Rane S, Lypowy J, He M, Chen IY, Vashistha H, Yan L, Malhotra A, Vatner D, Abdellatif M. MicroRNA-21 targets sprouty2 and promotes cellular outgrowths. Mol Bio Cell, 2008, 19: 3272-3282

37 Selcuklu SD, Donoghue MT, Spillane C. miR-21 as a key regulator of oncogenic processes. Biochem Soc Trans, 2009, 37: 918-925

38 Miyaki S, Sato T, Inoue A, Otsuki S, Ito Y, Yokoyama S, Kato Y, Takemoto F, Nakasa T, Yamashita S, Takada S, Lotz MK, Ueno-Kudo H, Asahara H. MicroRNA-140 plays dual roles in both cartilage development and homeostasis. Genes Dev, 2010, 24: 1173-1185

39 Herrera BM, Lockstone HE, Taylor JM, Ria M, Barrett A, Collins S, Kaisaki P, Argoud K, Fernandez C, Travers ME, Grew JP, Randall JC, Gloyn AL, Gauguier D, McCarthy MI, Lindgren CM. Global microRNA expression profiles in insulin target tissues in a spontaneous rat model of type 2 diabetes. Diabetologia, 2010, 53: 1099-1109

40 Liu SP, Fu RH, Yu HH, Li KW, Tsai CH, Shyu WC, Lin SZ. MicroRNAs regulation modulated self-renewal and lineage differentiation of stem cells. Cell Transplant, 2009, 18: 1039-1045

41 Baraniskin A, Birkenkamp-Demtroder K, Maghnouj A, Zöllner H, Munding J, Klein-Scory S, Reinacher-Schick A, Schwarte-Waldhoff
I, Schmiegel W, Hahn SA. miR-30a-5p suppresses tumor growth in colon carcinoma by targeting DTL. Carcinogenesis, 2012, 33: 732-739

42 Buechner J, Tømte E, Haug BH, Henriksen JR, Løkke C, Flægstad T, Einvik C. Tumour-suppressor microRNAs let-7 and mir-101 target the proto-oncogene mycn and inhibit cell proliferation in MYCNamplified neuroblastoma. Br J Cancer, 2011, 105: 296-303

43 Kottakis F, Polytarchou C, Foltopoulou P, Sanidas I, Kampranis SC, Tsichlis PN. FGF-2 regulates cell proliferation, migration, and angiogenesis through an NDY1/KDM2B-MiR-101-EZH2 pathway. Cell Molecular, 2011, 43: 285-298

44 Singh SK, Kagalwala MN, Parker-Thornburg J, Adams H, Majumder $\mathrm{S}$. Rest maintains self-renewal and pluripotency of embryonic stem cells. Nature, 2008, 453: 223-227

45 Georgantas RW 3rd, Hildreth R, Morisot S, Alder J, Liu CG, Heimfeld S, Calin GA, Croce CM, Civin CI. CD34 ${ }^{+}$hematopoietic stem-progenitor cell microRNA expression and function: A circuit diagram of differentiation control. Proc Natl Acad Sci USA, 2007, 104: 2750-2755

46 Phillips Bt, Gassei K, Orwig Ke. Spermatogonial stem cell regulation and spermatogenesis. Philos Trans R Soc Lond B Biol Sci, 2010, 365: 1663-1678

Open Access This article is distributed under the terms of the Creative Commons Attribution License which permits any use, distribution, and reproduction in any medium, provided the original author(s) and source are credited.

\section{Supporting Information}

Figure S1 Significantly differentially expressed miRNAs between of type B spermatogonia cells (BSc) and primary spermatocytes (PSc) validated by stem-loop RT-PCR experiments.

Table S1 Statistics of deep-sequencing data of small RNA libraries of BSc and PSc.

Table S2 Expression levels of all detected miRNAs and miRNAs expressed at extremely high levels in either BSc or PSc.

Table S3 All differentially expressed miRNA and their corresponding target genes.

Table S4 Primers for stem-loop real-time PCR of 6 marker miRNAs.

The supporting information is available online at life.scichina.com and link.springer.com. The supporting materials are published as submitted, without typesetting or editing. The responsibility for scientific accuracy and content remains entirely with the authors. 